\section{III.12 \\ The Emergency Observation Ward in Mass-Casualty Situations}

M.K.F. Leong, V. Anantharaman

Accident \& Emergency Department

Singapore General Hospital, Singapore

Mass-casualty situations often stress emergency departments to the point of decompensation and chaos. Emergency observation units can help to buffer this stress.

In March 1994, 59 Japanese, Korean, and Chinese tourists developed severe gastroenteritis soon after breakfast in their hotel. The emergency department (ED) of Singapore General Hospital, the largest acute-care, tertiary hospital in Singapore, received 49 casualties. The department, which attends to about 170,000 patients annually, had two immediate problems-very short notice to cope with the sudden influx of acutely ill patients, and the language barrier between patients and medical staff. The emergency observation ward (EOW) was mobilized. It was organized to cater to consultation, documentation, treatment, and recovery. This enabled the initial chaos to move into a smooth, organized factory-line flow system, allowing optimal delivery of patient care. Mobilization of appropriate medical and ancillary staff also facilitated the process. Within seven hours, 53 patients recovered well enough for discharge. Six required further in-patient treatment in the main hospital.

The EOW proved to be a valuable asset to the $\mathrm{ED}$ in this mass-casualty accident. With very short notice, it could be reorganized to absorb the sudden surge in demand for emergency care.

\section{III.13}

\section{Thyrotropin Releasing Hormone Affects the Duration of Propofol Anesthesia}

L.O.D. Koskinen, $M D$, PhD, J.E. Larsson, $M D$, G. Wahlström, $M D, P h D$ Department of NBC Defense, National Defense Research Establishment, Sweden, and the Department of Neurosurgery University Hospital Umeå Dept. of Pharmacology

Umeå University, Sweden

The neuropeptide thyrotropin-releasing hormone (TRH) elicits several nonhormonal effects, including blood-pressure effects, cerebrovasodilation, and analepsis. Beneficial effects in a variety of trauma models and shock states have been shown. The recently introduced intravenous anesthetic propofol is used increasingly in intensive-care medicine. Due to the analeptic effect of TRH, it was of interest to study the effect of the peptide on the induction and duration of propofol anesthesia in rats.

The rats were anesthetized by an IV infusion of propofol (10 $\mathrm{mg} / \mathrm{kg} / \mathrm{min}$ ) during continuous EEG monitoring. As endpoint for induction, a burst suppression period of one second or longer was used. The duration of anesthesia was determined as the time elapsed from the end-point to the regain of righting. The concentration of propofol in different brain regions was determined using HPLC.
Thyrotropin releasing hormone pretreatment $(5 \mathrm{mg} / \mathrm{kg}, \mathrm{n}=$ 6) had no significant effect on the induction dose of propofol as compared with controls pretreated with saline $(n=6)$. However, administration of TRH $(n=7)$ immediately after induction reduced the duration of anesthesia by $30.1 \pm 6.7 \%$ (mean \pm SEM) as compared with controls $(n=7)$. The propofol concentration at the time of recovery was significantly higher in the brain stem, cerebellum, hippocampus, and striatum in the animals treated with TRH after induction as compared with controls.

Thus, TRH reduces the duration of anesthesia induced by propofol. This effect seems to be due to a pharmacodynamic modification. This is to be considered in studies evaluating the effects of TRH in trauma and shock states. 